\title{
Serum biomarkers and clinical outcomes in heart failure patients treated de novo with carvedilol
}

\author{
Jadwiga Nessler ${ }^{1}$, Bohdan Nessler ${ }^{1}$, Renata Golebiowska-Wiatrak ${ }^{1}$, Ilona Palka ${ }^{1}$, \\ Andrzej Gackowski ${ }^{1}$, Mariusz Kitlinski ${ }^{2}$, Olle Melander ${ }^{3}$, Artur Fedorowski ${ }^{2,3}$ \\ ${ }^{1}$ Department of Coronary Disease, Institute of Cardiology, Jagiellonian University, \\ John Paul II Hospital, Cracow, Poland \\ ${ }^{2}$ Department of Cardiology, Skane University Hospital, Malmo, Sweden \\ ${ }^{3}$ Department of Clinical Sciences, Clinical Research Center, Lund University, \\ Skane University Hospital, Malmo, Sweden
}

\begin{abstract}
Background: The role of inflammatory and hemodynamic stress biomarkers in heart failure (HF) patients treated de novo with beta-blockers has been poorly studied.
\end{abstract}

Methods: A total of 86 patients (age $56 \pm 9$ years, 81 men) with left ventricular ejection fraction $(L V E F)<40 \%$ and previously not treated with beta-blockers were initiated on carvedilol. At baseline and 12 months later we performed echocardiography, cardiopulmonary exercise testing, and determined serum levels of B-type natriuretic peptide (BNP), endothelin-1 (ET-1), $C$-reactive protein (CRP), interleukin-6, and tumor necrosis factor alpha (TNF- $\alpha$ ). Patients were followed up over a total period of $9 \pm 3$ years from baseline.

Results: Increased baseline CRP and its on-treatment decrease were associated with improvement of LVEF (est. coefficient per one SD: 1.6; 95\% CI: $-0.05,3.28 ; p=0.056$, and -1.80; $-3.43,-0.18 ; p=0.030$, respectively) and diminishing of $L V$ end-systolic volume index $\left[m L / m^{2}\right](-6.83 ;-11.32 ;-2.34 ; p=0.003$, and $5.85 ; 1.23 ;-10.46 ; p=0.014$, respectively). Higher baseline ET-1 and on-treatment increase in TNF- $\alpha$ predicted frequent admissions (> 1) for cardiac complications (odds ratio per one SD: 1.98; 95\% CI: 1.09-3.59; $p=0.025$, and 2.07, 1.12-3.84, $p=0.021$, respectively) whereas higher baseline BNP was asociated with increased mortality (hazard ratio per one SD: 2.09, 95\% CI: 1.26-3.45; $p=0.004$ ).

Conclusions: Serum biomarkers may have different roles in prediction of clinical outcomes among HF patients treated de novo with carvedilol. (Cardiol J 2013; 20, 2: 144-151)

Key words: heart failure, beta-blocker, biological markers, natriuretic peptides, endothelin-1, C-reactive protein

\section{Introduction}

Effective treatment of chronic heart failure (CHF) has become a constant challenge for clinicians in the developed countries [1]. One of the therapeutic keystones in CHF, along with angiotensin-converting enzymes inhibitors (ACEI), is use of beta-blockers, which improves both left ventricular (LV) function and survival [2]. In recent years, increased attention has been focused on

Address for correspondence: Artur Fedorowski, Arrhythmia Department, Inga Marie Nilssons gata 46, Skåne University Hospital, 20502 Malmö, Sweden, tel: +46 4033 10 00, fax: +46 403912 22, e-mail: artur.fedorowski@med.lu.se 
various circulating biologically active substances, collectively known as plasma biomarkers, and their utility in HF prognosis and therapy monitoring [3]. Some of them, such as natriuretic peptides, have already entered clinical practice, and others, such as endothelin-1 (ET-1) or C-reactive protein (CRP), are still under evaluation [2, 3]. Hitherto, only a few studies have been performed to assess the predictive role of biomarkers and their on-treatment changes among CHF patients treated with beta-blockers, and specifically with carvedilol. Although carvedilol tends to reduce plasma levels of biomarkers such as CRP [4] or B-type natriuretic peptide (BNP) [5], the potential clinical implication of higher vs. lower initial biomarker levels and their responses to treatment have not been satisfactorily explored.

Consequently, we proposed to investigate a selection of inflammatory and haemodynamic stress biomarkers at the initiation and during carvedilol treatment in the population of symptomatic CHF patients. The aim of this study was to evaluate whether recently introduced biomarkers can be used in prediction of therapeutic response, risk of CHF exacerbation and long-term mortality.

\section{Methods}

\section{Study population}

The study population consisted of 86 patients (81 men, mean age $56 \pm 9$ years) with $\mathrm{CHF}$ who met the following inclusion criteria: functional capacity according to NYHA class II or III, left ventricular ejection fraction (LVEF) $<40 \%$, and no beta-blocker treatment for at least 3 months before entering the study. Patients were recruited in the years 2000-2001. The overall study design is shown in Figure 1. We excluded patients who had undergone percutaneous transluminal coronary angioplasty (PTCA) or coronary artery bypass grafting (CABG) within 3 months; with significant aortic or mitral valve defects except functional mitral regurgitation related to HF; with glomerular filtration rate below $60 \mathrm{~mL} / \mathrm{min}$; and with acute or chronic systemic disorders. All patients were receiving ACE (1 patient was on angiotensin receptor blocker [ARB]) at baseline and some were also treated with diuretics and/or digoxin. Carvedilol was introduced and titrated according to the regimen previously presented [6], starting with a dose of $3.125 \mathrm{mg}$ twice a day for 2 weeks.

The Ethics Committee of the Jagiellonian University accepted the study protocol and all patients gave their informed consent. In particu-

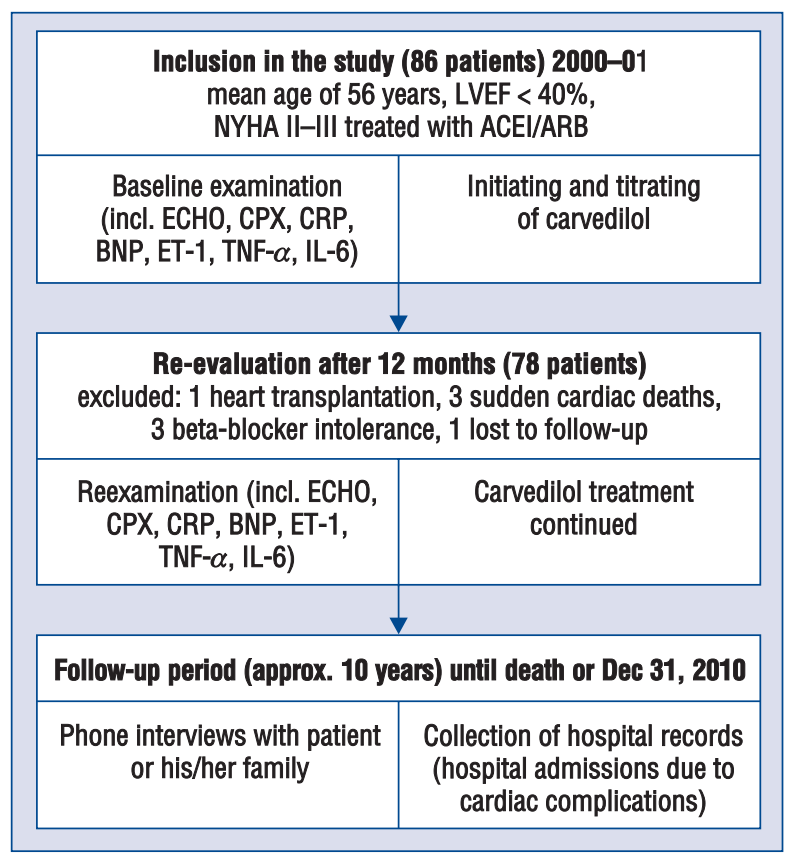

Figure 1. The overall study design; abbreviation - see the text.

lar, we decided to have a 3-month period without beta-blockers before starting carvedilol treatment to evaluate a net effect of carvedilol on the study endpoints. All the patients were informed that we abstained from beta-blockers for 3 months and the Ethics Committee accepted our rationale for the specific study design.

\section{Baseline examination and follow-up}

The following parameters were assessed at the start of the trial and after three and twelve months of carvedilol treatment; cardiac dimensions and function on echocardiography; mean heart rate at 24 hour ambulatory ECG monitoring; exercise capacity on cardiopulmonary stress test (CPX); and serum concentrations of BNP, ET-1, CRP, interleukin-6 (IL-6), and tumor necrosis factor alpha (TNF- $\alpha$ ).

The protocol for echocardiographic measurements has been described in detail previously [7]. Briefly, 2D-echocardiography was performed to calculate left ventricular end-diastolic and end-systolic volumes (LVEDV and LVESV) adjusted for body surface (as a respective index value). LVEF was calculated using the modified Simpson's method, averaging three consecutive measurements. These assessments are generally accepted as quantitative tools for evaluation of drug effects on ventricular remodeling in patients with reduced ejection fraction [8]. CPX was performed, as 
described in detail previously [6], according to the modified Naughton protocol as recommended by European Society of Cardiology [9]. Peak oxygen consumption expressed in $\mathrm{mL} / \mathrm{kg} / \mathrm{min}\left(\mathrm{VO}_{2}\right.$ peak $)$ and as a percentage of the calculated normal value $\left(\mathrm{VO}_{2}\right.$ peak $\left.\% \mathrm{~N}\right)$ as well as metabolic equivalents (METs) value were recorded [10]. Serum levels of neurohormones and inflammatory markers were measured in blood drawn from the antecubital vein after 30 min supine rest at fasting state in the morning. Measurements were done at the Laboratory of Radioligands in Cracow and at the Biochemical Laboratory of John Paul II Hospital in Cracow. The following commercially available reagents were used in the immunoenzymatic method (normal range in parenthesis): Immuno-Biological Laboratories (Hamburg) for BNP (up to $100 \mathrm{pg} / \mathrm{mL}$ ) and TNF- $\alpha$ (up to $5.0 \mathrm{pg} / \mathrm{mL}$ ), Milenia for IL-6 (up to $5.0 \mathrm{pg} / \mathrm{mL}$ ) and Cayman for ET-1 (up to $3.0 \mathrm{pg} / \mathrm{mL}$ ). Serum CRP was measured enzymatically using monoclonal anti-CRP antibodies labeled with horse-radish peroxidase (normal range up to $10 \mathrm{mg} / \mathrm{L}$ ).

The treatment with carvedilol was continued after 12 months with a mean dose of $25.8 \pm 15.2 \mathrm{mg} /$ /day. All patients were followed up over a total period of $9 \pm 3$ years from baseline until December 31,2010 or death. Information on the vital status of participant and the date of death if applicable was systematically collected by phone interviews with the patient or his/her family according to a special questionnaire. In parallel, data on a total number of heart-related hospitalizations (CHF exacerbations or coronary events) was systematically updated before the end of follow-up.

\section{Statistical analysis}

Temporal changes in continuous variables were compared using paired T-test. As serum concentrations of studied biomarkers were right skewed, they were $\log$ transformed prior to analyses. All biomarkers were scaled to one standard deviation (SD) of log-value. A linear regression model adjusted for age and gender was applied to assess relations between log-transformed baseline biomarkers levels and their changes during follow-up with improvement of LV function and exercise capacity parameters (derived from echocardiography [ECHO] and CPX, respectively). Similarly, a logistic regression model adjusted for age and gender was used to analyze relations between biomarkers and frequent hospital admissions due to cardiac complications, defined as a binary variable of more than one hospitalization due to CHF exacerbation or coronary event during follow-up period. The Kaplan-Maier method and multivariate-adjusted (for age and gender) Cox regression model were applied to assess biomarkers association with long-term mortality. All analyses were performed using IBM SPSS statistical software version 19.0 for Windows (SPSS Inc., Chicago, IL). All tests were two-sided and $\mathrm{p}<0.05$ was considered statistically significant.

\section{Results}

Baseline characteristics of the study population are summarized in Table 1. Twenty-seven (31\%) patients were in NYHA class II, and the rest in NYHA class III. In terms of etiology, 64 (74\%) patients had ischemic heart disease, of these 61 (71\%) had suffered prior myocardial infarction. Seventy-three (85\%) patients were at sinus rhythm at the start of study. Carvedilol was titrated to the mean dose of $25 \pm 14 \mathrm{mg} /$ daily during first 3 months after initiation of therapy. Mean doses of previously prescribed medications did not change significantly within 1 -year follow-up. Between the $3^{\text {rd }}$ and $12^{\text {th }}$ month, 1 patient underwent heart transplantation, 3 sudden cardiac deaths occurred, carvedilol was discontinued in 3 patients because of sinus bradycardia $(\mathrm{n}=2)$ or worsening of $\mathrm{CHF}(\mathrm{n}=1)$, and 1 patient was lost to follow-up. Therefore, the final analysis at 12 months was done in 78 patients.

The temporal changes in the clinical parameters and assessed plasma biomarkers are presented in Table 2, Figure 2A, and 2B, respectively. As can be seen in Table 3 , both baseline CRP $\left(\mathrm{CRP}_{0}\right)$ and its on-treatment decrease $(\triangle \mathrm{CRP})$ were predictive of LV function improvement $(\triangle \mathrm{LVEF} \%$ and $\triangle$ LVESVI). This association was independent of baseline LVEF $\left(\mathrm{LVEF}_{0}\right)$ and LVESVI $\left(\mathrm{LVESVI}_{0}\right)$. After additional adjustment for $\mathrm{LVEF}_{0}$ both $\mathrm{CRP}_{0}$ and $\triangle \mathrm{CRP}$ were still associated with improvement of LVEF (estimate coefficient per one SD: 1.6; 95\% confidence interval $[95 \% \mathrm{CI}]:-0.05 ; 3.28$; $\mathrm{p}=0.056$, and $-1.80 ;-3.43,-0.18 ; \mathrm{p}=0.030$, respectively) as they were in relation to $\triangle$ LVESVI after adjustment for its initial value $(-6.83$; $-11.32 ;-2.34 ; \mathrm{p}=0.003$, and $5.85 ; 1.23-10.46$; $\mathrm{p}=0.014$, respectively). In contrast, controlling for $\mathrm{LVESVI}_{0}$ and $\mathrm{LVEDVI}_{0}$ distinctly attenuated relationship between on-treatment reduction of TNF- $\alpha$ and LV parameters (non significant, data not shown), whereas trends for $\Delta \mathrm{BNP}, \Delta \mathrm{IL}-6$ and $\mathrm{LV}$ function improvement were not independent of baseline LV function parameters (data not shown). Moreover, as can be further noticed in Table 3, there was a weak 
Table 1. Baseline characteristics, biomarkers concentration and pharmacological treatment of study participants $(n=8)$.

\begin{tabular}{|c|c|}
\hline Characteristic & $\begin{array}{l}\text { Mean } \pm \text { SD or } \\
\text { percentage } \\
\text { (number) }\end{array}$ \\
\hline Age [years] & $56 \pm 9$ \\
\hline Gender [\%, men] & $84(81)$ \\
\hline BMI $\left[\mathrm{kg} / \mathrm{m}^{2}\right]$ & $27 \pm 4$ \\
\hline $\mathrm{SBP}[\mathrm{mm} \mathrm{Hg}]$ & $127 \pm 15$ \\
\hline $\mathrm{DBP}[\mathrm{mm} \mathrm{Hg}]$ & $84 \pm 8$ \\
\hline Heart rate $[\mathrm{bpm}]$ & $86 \pm 18$ \\
\hline Hypertension [\%] & $69(59)$ \\
\hline Diabetes [\%] & $17(15)$ \\
\hline Hypercholesterolemia [\%] & $62(53)$ \\
\hline Total cholesterol [mg/dL] & $215 \pm 44$ \\
\hline HDL cholesterol [mg/dL] & $42 \pm 11$ \\
\hline LDL cholesterol [mg/dL] & $136 \pm 35$ \\
\hline Hemoglobin $[\mathrm{g} / \mathrm{dL}]$ & $14 \pm 1$ \\
\hline Creatinine clearance $[\mathrm{mL} / \mathrm{min}]$ & $102 \pm 35$ \\
\hline \multicolumn{2}{|l|}{ Biomarkers: } \\
\hline CRP [mg/L] & $14.4 \pm 14.3$ \\
\hline Interleukin-6 [pg/mL] & $9.4 \pm 8.5$ \\
\hline TNF- $\alpha[p g / m L]$ & $12.2 \pm 8.7$ \\
\hline BNP [pg/mL] & $464 \pm 215$ \\
\hline Endothelin-1 [pg/mL] & $49 \pm 95$ \\
\hline \multicolumn{2}{|l|}{ Pharmacological treatment: } \\
\hline ACE inhibitor & $99(85)$ \\
\hline ARB & $1(1)$ \\
\hline Loop diuretic & $49(42)$ \\
\hline Spironolactone & $71(61)$ \\
\hline Digitalis & $6(5)$ \\
\hline ASA & $77(66)$ \\
\hline Statin & $62(53)$ \\
\hline
\end{tabular}

$\mathrm{BMI}$ - body-mass index; SBP — systolic blood pressure; DBP — diastolic blood pressure; Hypercholesterolemia, total serum cholesterol $\geq 200 \mathrm{mg} / \mathrm{dL}$; CRP - C-reactive protein; TNF- $\alpha$ - tumor necrosis factor- $\alpha$; BNP - B-type natriuretic peptide; ACE - angiotensin converting enzyme; ARB angiotensin receptor blocker; ASA — acetylsalicylic acid

association of higher baseline ET-1 (ET- $\left.1_{0}\right)$ and its on-treatment decrease $(\Delta \mathrm{ET}-1)$ with the improvement of $\mathrm{VO}_{2 \text { peak }}$, markedly attenuated after adjustment for initial $\mathrm{VO}_{2 \text { peak }}$ value $(0.60 ;-0.24 ; 1.44$; $\mathrm{p}=0.16$, and $-0.48 ;-1.30 ; 0.34 ; \mathrm{p}=0.24$, respectively).
Data on heart-related hospital admissions were available in 73 patients (mean \pm SD: $1.9 \pm$ \pm 1.8 ; range $0-10$ ); of these 13 patients had no hospitalization at all, and 25 patients were admitted only once during the follow-up period. Higher ET- $1_{0}$ (odds ratio [OR] per one SD: $1.98 ; 95 \%$ CI: $1.09-3.59 ; \mathrm{p}=0.025)$ and increase in TNF- $\alpha$ $(\Delta \mathrm{TNF}-\alpha)$ at 12 -month follow-up $(2.07 ; 1.12-3.84$; $\mathrm{p}=0.021$ ) were independent predictors of frequent admissions for cardiac complications.

Mortality data were available in 83 patients; of these $33(38.4 \%)$ died after a mean time of 6 years (range 1-11 years). In the Cox regression analysis, none of baseline clinical parameters significantly differed between those patients who died and those who survived but there was a trend for association between lower LVEF\% and higher mortality (hazard ratio [HR] per one percent decrease of LVEF, 95\% CI: 1.04 ; 0.99-1.10; $\mathrm{p}=0.11$ ). Among studied biomarkers, higher baseline level of $\mathrm{BNP}\left(\mathrm{BNP}_{0}\right)$ was a strong predictor of mortality $(\mathrm{n}=76$, HR per one SD: $2.09 ; 1.26-3.45 ; \mathrm{p}=0.004)$ but on-treatment change in BNP was not associated with mortality $(0.86$; 0.55 $-1.34 ; \mathrm{p}=0.51)$. In parallel, on-treatment increase in TNF- $\alpha$ indicated a trend toward higher mortality (HR: $1.44 ; 0.97-2.14 ; \mathrm{p}=0.069)$. Relationship between BNP level on entering the study and long-term mortality is illustrated in Figure 3, which shows Kaplan-Maier survival curves for study population stratified by mean baseline value of BNP ( $446 \mathrm{pg} / \mathrm{mL})$. Study participants in the higher $\mathrm{BNP}_{0}$ stratum demonstrated two-fold increased mortality as compared with participants in the lower stratum of $\mathrm{BNP}_{0}(50 \%$ vs. $25 \%$ ).

\section{Discussion}

We report here that cardiovascular plasma biomarkers may relate to different clinical outcomes in a population of $\mathrm{HF}$ patients treated with carvedilol. Amelioration of echocardiographic LV parameters and exercise tolerance during carvedilol treatment was expected [11-13] as was also decrease in circulating inflammatory and vasoactive substances $[4,14,15]$. As summarized in Table 4 , increased $\mathrm{BNP}_{0}$ predicted total long-term mortality; whereas increased ET- $1_{0}$ indicated patients who were at higher risk of frequent hospitalizations as did also on-treatment increase in TNF- $\alpha$. Moreover, higher ET- $1_{0}$ and its on-treatment decrease tended to be associated with improvement of exercise capacity on CPX, although not significantly. In parallel, higher $\mathrm{CRP}_{0}$ was predictive of $\mathrm{LV}$ function improvement as 
Table 2. Changes in echocardiographic and exercise capacity parameters during treatment with carvedilol $(\mathrm{n}=78)$; mean $\pm \mathrm{SD}$.

\begin{tabular}{lcccc}
\hline Parameter & Baseline & At 3 months & At 12 months & P (0-12 months) \\
\hline LVEF $[\%]$ & $27 \pm 6$ & $34 \pm 8$ & $37 \pm 9$ & $<0.001$ \\
LVESVI $\left[\mathrm{mL} / \mathrm{m}^{2}\right]$ & $68 \pm 27$ & $63 \pm 28$ & $55 \pm 27$ & $<0.001$ \\
LVEDVI $\left[\mathrm{mL} / \mathrm{m}^{2}\right]$ & $97 \pm 39$ & $94 \pm 35$ & $86 \pm 33$ & 0.005 \\
$\mathrm{VO}_{2 \text { peak }}[\mathrm{mL} / \mathrm{kg} / \mathrm{min}]$ & $15 \pm 5$ & $15 \pm 4$ & $16 \pm 4$ & 0.10 \\
$\mathrm{VO}_{\text {2peak }} \% \mathrm{~N}$ & $52 \pm 16$ & $53 \pm 16$ & $57 \pm 15$ & 0.04 \\
$\mathrm{~T}_{\text {max }}[\mathrm{min}]$ & $9.9 \pm 4.6$ & $11.2 \pm 5.1$ & $12.4 \pm 4.7$ & $<0.001$ \\
METs & $4.3 \pm 2.3$ & $5.0 \pm 2.4$ & $5.5 \pm 2.1$ & $<0.001$ \\
24h-heart rate $[/ \mathrm{min}]$ & $76 \pm 11$ & $72 \pm 11$ & $72 \pm 10$ & $<0.001$ \\
\hline
\end{tabular}

LVEF - left ventricular ejection fraction; LVEDVI - left ventricular end-diastolic volume index; LVESVI — left ventricular end-systolic volume index; $\mathrm{VO}_{2 \text { peak }}$ - peak oxygen uptake; $\mathrm{VO}_{2 \text { peak }} \% \mathrm{~N}$ - peak oxygen uptake as \% of valid normal; $\mathrm{T}_{\max }$ - cardiopulmonary exercise test duration; MET — metabolic equivalent; $24 \mathrm{~h}$-heart rate — mean heart rate on 24-hour ambulatory ECG

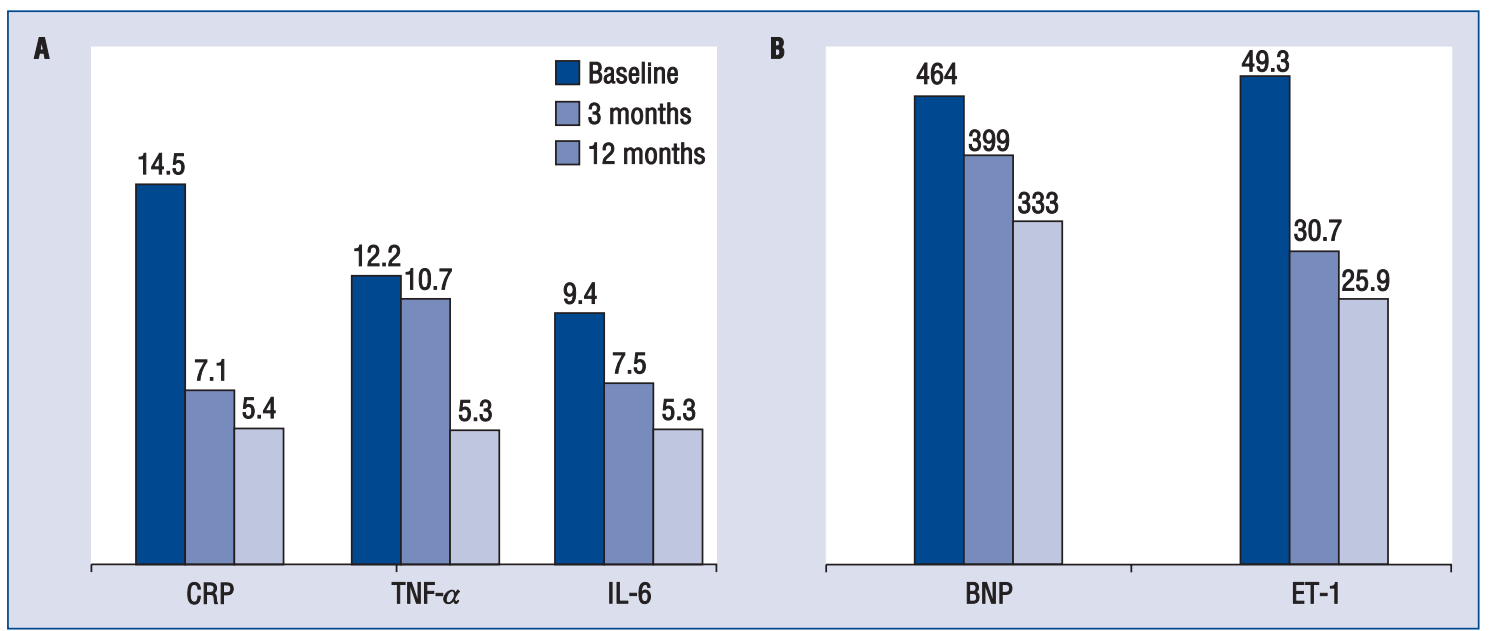

Figure 2. A. Changes in inflammatory biomarkers during 12-month therapy with carvedilol; B. Changes in hemodynamic stress biomarkers during 12-month therapy with carvedilol; CRP - C-reactive protein [mg/L]; TNF- $\alpha$ - tumor necrosis factor- $\alpha$ [pg/mL]; IL-6 — interleukin-6 [pg/mL]; BNP — B-type natriuretic peptide [pg/mL]; ET-1 — endothelin-1 $[\mathrm{pg} / \mathrm{mL}]$.

was also decrease in this biomarker concentration during first 12 months of therapy. So, although the total cardiac function and exercise tolerance improved and serum concentrations of the assessed plasma biomarkers significantly decreased there was a distinct diversity in biomarkers' predictive properties. In concordance with previous reports [16-18], baseline BNP level was a strong predictor of all-cause mortality but in contrast to them it was not prognostic of subsequent cardiovascular hospitalizations. Further, on-treatment changes in BNP had no role in prediction of mortality, hospitalizations, or therapeutic response to carvedilol. This observation is important in the light of current debate on use of BNP as a therapy monitoring tool in CHF. The guidelines admit that such application of natriuretic peptides is "less clearly established" [2] and that results of previous trials seem contradictory [19, 20]. Endothelin-1, another emerging haemodynamic stress biomarker, demonstrated association with frequent hospitalizations, but not with mortality, which is only partially concordant with recent studies [21]. Interestingly, temporal changes in TNF- $\alpha$ and not its pretreatment concentration were also associated with the risk of cardiac exacerbations: patients with increasing on-treatment TNF- $\alpha$ level were more likely to be hospitalized. The role of TNF- $\alpha$ in deterioration of CHF was previously reported [22] and our study confirms these findings. 
Table 3. Association of log-transformed biomarkers levels at baseline and their on-treatment changes (0-12 months) with improvement of echocardiographic and exercise capacity parameters in adjusted linear regression model (estimate coefficient; 95\% confidence interval). Estimate coefficients are presented per one standard deviation of log-transformed biomarker level or its on-treatment change $(n=78)$.

\begin{tabular}{|c|c|c|c|c|c|c|}
\hline Biomarker & $\begin{array}{c}\Delta \text { LVEF } \\
{[\%]}\end{array}$ & $\begin{array}{l}\Delta \text { LVESVI } \\
{\left[\mathrm{mL} / \mathrm{m}^{2}\right]}\end{array}$ & $\begin{array}{l}\triangle \mathrm{LVEDVI} \\
{\left[\mathrm{mL} / \mathrm{m}^{2}\right]}\end{array}$ & $\begin{array}{c}\Delta \mathrm{VO}_{\text {2peak }} \\
{[\mathrm{mL} / \mathbf{k g} / \mathrm{min}]}\end{array}$ & $\Delta \mathbf{V O}_{\text {2peak }} \% \mathbf{N}$ & $\triangle$ METs \\
\hline $\log \mathrm{CRP}_{0}$ & $\begin{array}{c}1.82 ; 0.15 ; 3.49 \\
P=0.034\end{array}$ & $\begin{array}{c}-5.96 ;-10.76 ;-1.16 \\
P=0.016\end{array}$ & $\begin{array}{c}-3.64 ;-11.81 ; 4.54 \\
P=0.38\end{array}$ & $\begin{array}{c}-0.44 ;-1.46 ; 0.59 \\
P=0.48\end{array}$ & $\begin{array}{c}-0.76 ;-4.72 ; 3.19 \\
P=0.70\end{array}$ & $\begin{array}{c}-0.21 ;-0.67,0.26 \\
P=0.38\end{array}$ \\
\hline$\Delta$ Log CRP & $\begin{array}{c}-1.85 ;-3.51 ;-0.19 \\
P=0.030\end{array}$ & $\begin{array}{c}4.28 ;-0.59 ; 9.16 \\
P=0.084\end{array}$ & $\begin{array}{c}5.65 ;-2.45 ; 13.76 \\
P=0.17\end{array}$ & $\begin{array}{c}-0.08 ;-1.14 ; 0.98 \\
P=0.88\end{array}$ & $\begin{array}{c}-0.28 ;-3.39 ; 3.96 \\
P=0.88\end{array}$ & $\begin{array}{c}-0.01 ;-0.50,0.48 \\
P=0.97\end{array}$ \\
\hline Log IL-6 ${ }_{0}$ & $\begin{array}{c}0.30 ;-1.40 ; 2.00 \\
P=0.73\end{array}$ & $\begin{array}{c}-0.48 ;-5.35 ; 4.39 \\
P=0.85\end{array}$ & $\begin{array}{c}-0.66 ;-8.68 ; 7.36 \\
P=0.87\end{array}$ & $\begin{array}{c}0.21 ;-0.79 ; 1.21 \\
P=0.68\end{array}$ & $\begin{array}{c}0.87 ;-2.97 ; 4.71 \\
P=0.65\end{array}$ & $\begin{array}{c}0.26 ;-0.20 ; 0.73 \\
P=0.26\end{array}$ \\
\hline$\Delta$ Log IL-6 & $\begin{array}{c}-1.74 ;-3.57 ; 0.09 \\
P=0.061\end{array}$ & $\begin{array}{c}2.33 ;-3.07 ; 7.73 \\
P=0.39\end{array}$ & $\begin{array}{c}0.66 ;-8.32 ; 9.63 \\
P=0.89\end{array}$ & $\begin{array}{c}-0.11 ;-1.18 ; 0.96 \\
P=0.83\end{array}$ & $\begin{array}{c}0.14 ;-3.97 ; 4.26 \\
P=0.95\end{array}$ & $\begin{array}{c}-0.13 ;-0.63 ; 0.37 \\
P=0.61\end{array}$ \\
\hline Log TNF- $\alpha_{0}$ & $\begin{array}{c}0.87 ;-0.89 ; 2.63 \\
P=0.33\end{array}$ & $\begin{array}{c}1.78 ;-3.33 ; 6.88 \\
P=0.49\end{array}$ & $\begin{array}{c}0.79 ;-7.59 ; 9.17 \\
P=0.85\end{array}$ & $\begin{array}{c}-0.03 ;-1.04 ; 0.98 \\
P=0.96\end{array}$ & $\begin{array}{c}0.14 ;-3.73 ; 4.01 \\
P=0.94\end{array}$ & $\begin{array}{c}-0.27 ;-0.74 ; 0.21 \\
P=0.26\end{array}$ \\
\hline$\Delta$ Log TNF- $\alpha$ & $\begin{array}{c}0.06 ;-1.77 ; 1.88 \\
P=0.95\end{array}$ & $\begin{array}{c}-5.71 ;-10.63 ;-0.79 \\
P=0.024\end{array}$ & $\begin{array}{c}-8.09 ;-15.05 ;-1.14 \\
P=0.023\end{array}$ & $\begin{array}{c}0.07 ;-0.55 ; 0.69 \\
P=0.83\end{array}$ & $\begin{array}{c}0.69 ;-1.70 ; 3.07 \\
P=0.57\end{array}$ & $\begin{array}{c}0.18 ;-0.11 ; 0.47 \\
P=0.21\end{array}$ \\
\hline $\log \mathrm{BNP}_{0}$ & $\begin{array}{c}0.30 ;-1.54,2.13 \\
P=0.75\end{array}$ & $\begin{array}{c}-2.65 ;-7.73,2.44 \\
P=0.30\end{array}$ & $\begin{array}{c}-1.58 ;-8.66,5.51 \\
P=0.66\end{array}$ & $\begin{array}{c}-0.26 ;-1.27,0.74 \\
P=0.60\end{array}$ & $\begin{array}{c}-0.98 ;-4.78 \\
P=0.61\end{array}$ & $\begin{array}{c}0.15 ;-0.32 ; 0.63 \\
P=0.52\end{array}$ \\
\hline$\Delta$ Log BNP & $\begin{array}{c}-1.63 ;-3.55 ; 0.30 \\
P=0.096\end{array}$ & $\begin{array}{c}5.01 ;-0.43 ; 10.45 \\
P=0.071\end{array}$ & $\begin{array}{c}4.23 ;-3.57 ; 12.02 \\
P=0.28\end{array}$ & $\begin{array}{c}-0.18 ;-1.33 ; 0.97 \\
P=0.76\end{array}$ & $\begin{array}{c}-0.62 ;-4.97 ; 3.73 \\
P=0.78\end{array}$ & $\begin{array}{c}-0.07 ;-0.60 ; 0.46 \\
P=0.80\end{array}$ \\
\hline Log ET-10 & $\begin{array}{c}0.65 ;-1.14 ; 2.43 \\
P=0.47\end{array}$ & $\begin{array}{c}-1.10 ;-6.28 ; 4.07 \\
P=0.67\end{array}$ & $\begin{array}{c}0.13 ;-8.19 ; 8.45 \\
P=0.98\end{array}$ & $\begin{array}{c}0.90 ;-0.11 ; 1.90 \\
P=0.080\end{array}$ & $\begin{array}{c}2.68 ;-1.21 ; 6.56 \\
P=0.17\end{array}$ & $\begin{array}{c}0.32 ;-0.16 ; 0.80 \\
P=0.19\end{array}$ \\
\hline$\Delta$ Log ET-1 & $\begin{array}{c}-1.35 ;-3.09 ; 0.39 \\
P=0.13\end{array}$ & $\begin{array}{c}-0.08 ;-5.57 ; 5.41 \\
P=0.98\end{array}$ & $\begin{array}{c}-0.71 ;-9.52 ; 8.10 \\
P=0.87\end{array}$ & $\begin{array}{c}-0.90 ;-1.87 ; 0.07 \\
P=0.067\end{array}$ & $\begin{array}{c}-2.70 ;-6.44 ; 1.04 \\
P=0.15\end{array}$ & $\begin{array}{c}-0.01 ;-0.48 ; 0.46 \\
P=0.96\end{array}$ \\
\hline
\end{tabular}

CRP - C-reactive protein; IL-6 — interleukin-6; TNF- $\alpha$ - tumor necrosis factor- $\alpha$; BNP — B-type natriuretic peptide; ET-1 - endothelin-1; LVEF — left ventricular ejection fraction; LVEDVI — left ventricular end-diastolic volume index; LVESVI — left ventricular end-systolic volume index; $\mathrm{VO}_{2 \text { peak }}$ - peak oxygen uptake; $\mathrm{VO}_{2 \text { peak }} \% \mathrm{~N}$ - peak oxygen uptake as \% of valid normal; MET — metabolic equivalent

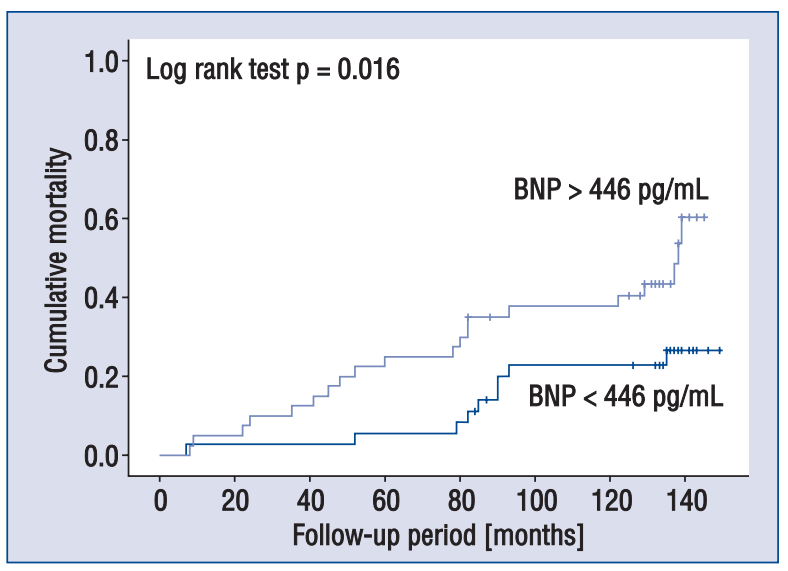

Figure 3. Kaplan-Maier survival curves for carvedilol-treated heart failure patients $(\mathrm{n}=76)$ dichotomized by mean initial B-type natriuretic peptide (BNP) value. All-cause mortality in the upper stratum (> $446 \mathrm{pg} / \mathrm{mL})$ was 2-fold increased as compared to the lower stratum $(<446 \mathrm{pg} / \mathrm{mL})$.

The most intriguing finding in the present study was a strong and independent predictive role of increased systemic inflammatory activity, as indicated by CRP, on carvedilol-induced impro-
Table 4. Potential role of different plasma biomarkers in prediction of clinical outcomes among carvedilol-treated heart failure patients based on the present study.

\begin{tabular}{lc}
\hline $\begin{array}{l}\text { Plasma } \\
\text { biomarker }\end{array}$ & Clinical outcome to predict \\
\hline $\mathrm{CRP}_{0}$ & LV function improvement \\
$\Delta \mathrm{CRP}$ & LV function improvement \\
$\mathrm{ET}-1_{0}$ & Hospitalization; Exercise capacity? \\
$\triangle \mathrm{ET}-1$ & Exercise capacity? \\
$\mathrm{BNP}_{0}$ & Long-term mortality \\
$\Delta \mathrm{TNF}-\alpha$ & Hospitalization; Mortality? \\
\hline
\end{tabular}

Abbreviations as in Tables 2 and 3

vement of $\mathrm{LV}$ function. In fact, CRP was the only plasma biomarker to show a consistent relationship with on-treatment changes in echocardiographic LV parameters. High-sensitivity CRP is an established cardiovascular risk factor in general, and for development of CHF in particular [23, 24]. Patients with CHF show activation of renin-angiotensin-aldosterone and the sympathetic nervous systems, which may lead to activation of proinflammatory 
cytokines but the exact mechanisms coupling CHF with systemic inflammation have not been hitherto explained [23]. Based on this study, it seems that HF patients with a relatively higher initial level of CRP benefit most from carvedilol, which may have a specific anti-inflammatory effect above and beyond the well-known antiadrenergic mechanism as suggested by previous studies [25]. Moreover, the on-treatment CRP reduction paralleled improvement of $\mathrm{LV}$ function making $\mathrm{CRP}$ an interesting potential biomarker for monitoring the therapeutic effect of carvedilol treatment. Taken together, in the pretreatment phase of carvedilol therapy the optimal biomarker selection would include BNP for evaluation of mortality risk, ET-1 for prognostic assessment of future cardiac exacerbations, and TNF- $\alpha$ for indentifying non-responders who are at high risk of frequent hospitalizations due to HF worsening. Further, CRP may be a valuable predictor of LV response both before and under the treatment. However, taking into account that carvedilol differs from other beta-blockers in its vasodilatory [26] and anti-inflammatory properties, these results cannot be extrapolated onto beta-blocker class in toto.

\section{Limitations of the study}

The study sample was relatively small and only a few women participated in the study. The open-label study design might potentially introduce a bias, especially in the clinical assessment of the patients. However, all the clinical evaluations as well as the laboratory measurements were performed in a blinded fashion by independent individuals. In addition, since beta-blockers are part of the standard treatment of patients with $\mathrm{CHF}$ and reduced LVEF, there was no control group without beta-blocker treatment. A significant part of the study sample had an ischemic HF etiology and, consequently, the role of inflammatory biomarkers (i.e. CRP) may differ in a population of HF patients without underlying ischemic heart disease. This group was underrepresented in this study and our results cannot be extrapolated without reservation on non-ischemic HF. Finally, data on smoking status were not available and we cannot exclude that a residual confounding in relation to inflammatory biomarkers existed due to this factor.

\section{Conclusions}

In conclusion, plasma biomarkers may have different roles in prediction of clinical outcomes among carvedilol-treated symptomatic HF patients.
Baseline BNP predicts long-term mortality, increased baseline CRP and its on-treatment decrease indicate higher probability of LV improvement, whereas higher baseline ET-1 and on-treatment increase in TNF- $\alpha$ identify those with higher risk of cardiac exacerbations during treatment. More studies on larger patients groups are needed to verify these findings.

\section{Acknowledgements}

This study was supported by grants from Collegium Medicum, Jagiellonian University, Cracow (501/KL/293, and 81/2002 from the central reserve for special projects financing).

O.M. and A.F. were supported by grants from the European Research Council (StG 282225), Swedish Medical Research Council, and the Swedish Heart and Lung Foundation.

Conflict of interest: none declared

\section{References}

1. Braunwald E. Shattuck lecture: Cardiovascular medicine at the turn of the millennium: Triumphs, concerns, and opportunities. N Engl J Med, 1997; 337: 1360-1369.

2. Dickstein K, Cohen-Solal A, Filippatos G et al. ESC Guidelines for the diagnosis and treatment of acute and chronic heart failure 2008: The Task Force for the Diagnosis and Treatment of Acute and Chronic Heart Failure 2008 of the European Society of Cardiology. Developed in collaboration with the Heart Failure Association of the ESC (HFA) and endorsed by the European Society of Intensive Care Medicine (ESICM). Eur Heart J, 2008; 29: 2388-2442.

3. Braunwald E. Biomarkers in heart failure. N Engl J Med, 2008; 358: 2148-2159.

4. Nagatomo Y, Yoshikawa T, Kohno T et al. Effects of beta-blocker therapy on high sensitivity c-reactive protein, oxidative stress, and cardiac function in patients with congestive heart failure. J Card Fail, 2007; 13: 365-371.

5. Fujimura M, Akaike M, Iwase T et al. Decrease in plasma brain natriuretic peptide level in the early phase after the start of carvedilol therapy is a novel predictor of long-term outcome in patients with chronic heart failure. Acta Cardiol, 2009; 64: 589-595.

6. Nessler J, Nessler B, Kitlinski M, Gackowski A, Piwowarska W, Stepniewski M. Concentration of BNP, endothelin 1, pro-inflammatory cytokines (TNF-alpha, IL-6) and exercise capacity in patients with heart failure treated with carvedilol. Kardiol Pol, 2008; 66: 144-151.

7. Nessler J, Nessler B, Kitlinski M, Stepniewski M, Piwowarska W. Restrictive left ventricular filling pattern and its effect on the clinical course of systolic heart failure in patients receiving carvedilol. Cardiol J, 2008; 15: 329-337.

8. Kramer DG, Trikalinos TA, Kent DM, Antonopoulos GV, Konstam MA, Udelson JE. Quantitative evaluation of drug or device effects on ventricular remodeling as predictors of therapeutic effects on mortality in patients with heart failure and reduced 
ejection fraction: A meta-analytic approach. J Am Coll Cardiol, 2010; 56: 392-406.

9. Cardiology WGoCREPaWGoHFotESo. Recommendations for exercise testing in chronic heart failure patients. Eur Heart J, 2001; 22: 37-45.

10. Balady GJ, Arena R, Sietsema K et al. Clinician's Guide to cardiopulmonary exercise testing in adults: A scientific statement from the American Heart Association. Circulation, 2010; 122: 191-225.

11. Metra M, Giubbini R, Nodari S, Boldi E, Modena MG, Dei Cas L. Differential effects of beta-blockers in patients with heart failure: A prospective, randomized, double-blind comparison of the long-term effects of metoprolol versus carvedilol. Circulation, 2000; 102: 546-551.

12. Palazzuoli A, Bruni F, Puccetti L et al. Effects of carvedilol on left ventricular remodeling and systolic function in elderly patients with heart failure. Eur J Heart Fail, 2002; 4: 765-770.

13. Lombardo RM, Reina C, Abrignani MG, Rizzo PA, Braschi A, De Castro S. Effects of nebivolol versus carvedilol on left ventricular function in patients with chronic heart failure and reduced left ventricular systolic function. Am J Cardiovasc Drugs, 2006; 6: 259-263.

14. Kawai K, Hata K, Takaoka H, Kawai H, Yokoyama M. Plasma brain natriuretic peptide as a novel therapeutic indicator in idiopathic dilated cardiomyopathy during beta-blocker therapy: A potential of hormone-guided treatment. Am Heart J, 2001; 141: 925-932.

15. Baran D, Horn EM, Hryniewicz K, Katz SD. Effects of beta-blockers on neurohormonal activation in patients with congestive heart failure. Drugs, 2000; 60: 997-1016.

16. Hartmann F, Packer M, Coats AJ et al. Prognostic impact of plasma N-terminal pro-brain natriuretic peptide in severe chronic congestive heart failure: A substudy of the Carvedilol Prospective Randomized Cumulative Survival (COPERNICUS) trial. Circulation, 2004; 110: 1780-1786.

17. Rosenberg J, Gustafsson F, Remme WJ, Riegger GA, Hildebrandt PR. Effect of beta-blockade and ACE inhibition on B-type natriuretic peptides in stable patients with systolic heart failure. Cardiovasc Drugs Ther, 2008; 22: 305-311.

18. Neuhold S, Huelsmann M, Strunk G et al. Prognostic value of emerging neurohormones in chronic heart failure during optimization of heart failure-specific therapy. Clin Chem, 2010; 56: 121-126.

19. Hunt SA, Abraham WT, Chin MH et al. 2009 focused update incorporated into the ACC/AHA 2005 Guidelines for the Diagnosis and Management of Heart Failure in Adults: A report of the American College of Cardiology Foundation/American Heart Association Task Force on Practice Guidelines: Developed in collaboration with the International Society for Heart and Lung Transplantation. Circulation, 2009; 119: e391-479.

20. Bohm M, Voors AA, Ketelslegers JM et al. Biomarkers: Optimizing treatment guidance in heart failure. Clin Res Cardiol, 2011; 100: 973-981.

21. Tang WH, Shrestha K, Martin MG et al. Clinical significance of endogenous vasoactive neurohormones in chronic systolic heart failure. J Card Fail, 2010; 16: 635-640.

22. Somasundaram P, Sung BH, Mousa SA, Wilson MF. Acute congestive heart failure and tumor necrosis factor alpha. Am J Geriatric Cardiol, 2000; 9: 76-80.

23. Osman R, L'Allier PL, Elgharib N, Tardif JC. Critical appraisal of C-reactive protein throughout the spectrum of cardiovascular disease. Vasc Health Risk Manag, 2006; 2: 221-237.

24. Smith JG, Newton-Cheh C, Almgren P et al. Assessment of conventional cardiovascular risk factors and multiple biomarkers for the prediction of incident heart failure and atrial fibrillation. J Am Coll Cardiol, 2010; 56: 1712-1719.

25. Yue TL, Cheng HY, Lysko PG et al. Carvedilol, a new vasodilator and beta adrenoceptor antagonist, is an antioxidant and free radical scavenger. J Pharmacol Exp Ther, 1992; 263: 92-98.

26. Ruffolo RR, Jr., Gellai M, Hieble JP, Willette RN, Nichols AJ. The pharmacology of carvedilol. Eur J Clin Pharmacol, 1990; 38 (suppl. 2): S82-S88. 